\title{
A Novel Histogram Definition for Fuzzy Color Spaces
}

\author{
J. Chamorro-Martínez, D. Sánchez, and J.M. Soto-Hidalgo
}

\begin{abstract}
In this paper we introduce fuzzy naturals-based histograms on fuzzy color spaces. In our approach, histograms are fuzzy probability distributions on a fuzzy color space, where the fuzzy probability is calculated as the quotient between a fuzzy natural number and the number of pixels in the image, the former being a fuzzy (non-scalar) cardinality of a fuzzy set. This approach to histograms avoids the well-known disadvantages of the ordinary sigma-count as an estimation of the probability. We illustrate the potential application of the proposal by applying it to the problem of dominant color selection.
\end{abstract}

\section{INTRODUCTION}

Color is a powerful feature for image analysis and description. In color images, each pixel consists of three (or more) components corresponding to the primary colors in a given color space (e.g, red, green and blue for RGB). Color image techniques operate using the information given by these components, avoiding limitations of those methods which only use grey level data.

In some applications it is very important to take into account the particular perception of colors of a certain user when processing an image. However, the perception of colors of humans is not that of a triplet of real values. This problem has several facets:

- In practice, humans are able to distinguish a remarkably lesser amount of numbers that computers, despite the color space employed.

- Humans group together those colors that are indistinguishable for them. However, this grouping is imprecise and not homogeneous, i.e., triplets are not uniformly distributed among groups (corresponding to perceived colors), and the boundaries between these perceived colors are not precise.

- The previous facets may vary depending on the particular user and/or the context or application.

In order to incorporate the particular, imprecise perception of colors by the user and/or for certain applications, several authors have proposed to employ fuzzy sets [11], [12], [14]. In this approach, colors in an image are linguistic labels represented as fuzzy subsets of triplets of a certain color space [22], [25]. This is important in order to determine the semantic interpretation of colors for human-machine interaction, or to take into account the similarity between colors in a color space as perceived by the user [21].

J. Chamorro-Martínez and D. Sánchez are with the Department of Computer Science and Artificial Intelligence, University of Granada, Spain. J.M. Soto-Hidalgo is with the Department of Computer Architecture, Electronics and Electronic Technology, University of Córdoba, Spain. email:\{jesus,daniel\}@decsai.ugr.es,jmsoto@uco.es

This work has been partially supported by the Spanish Government under the TEC2006-13845 and Consolider Ingenio 2010 MIPRCV (CSD200700018) projects.
In the scope of image analysis and processing, the histogram is the basis for numerous techniques for image restoration, enhancement, segmentation, retrieval, etc [19]. The histogram of an image is a function $h\left(\mathbf{c}_{\mathbf{k}}\right)=n_{k}$ where $\mathbf{c}_{\mathbf{k}}=[x, y, z]$ is a color and $n_{k}$ is the number of pixels in the image having the color $\mathbf{c}_{\mathbf{k}}$. It is common to normalize a histogram by dividing each of its values by the total number of pixels, obtaining an estimate of the probability of occurrence of a color $\mathbf{c}_{\mathbf{k}}$.

Working with fuzzy colors suggests to extend the notion of histogram to "fuzzy histogram" in order to manage the imprecision in (i) color description by means of fuzzy colors and, consequently, in (ii) fuzzy color counting. In this sense, a fuzzy histogram will give us information about the frequency of each fuzzy color.

In the literature there are several proposals which define histograms over a set of fuzzy colors [7], [10], [17], [18]. One drawback of most of these proposals is that they work only with intensities. In addition, the counting of fuzzy colors is performed by using the sigma-count (i.e., the sum of membership degrees). However, the sigma-count is not a suitable measure of cardinality in many applications, as it has been recognized by several authors [9], [13]. Proposals based on the sigma-count summarize the counting in a single number, so they do not represent the imprecision of the count itself.

To solve the problems above, after introducing a formalization of the concept of fuzzy color space, in this paper we propose to "count" the occurrences of a given fuzzy color by means of the new definition of fuzzy natural numbers introduced in [5]. In this definition, a natural number is represented by means of a probability distribution on $\mathbb{N}$. On the basis of these fuzzy naturals, a new definition of fuzzy frequency is proposed.

The rest of the paper is organized as follows. Section II formalize the concepts of fuzzy color and fuzzy color space. In section III the fuzzy natural numbers and fuzzy frequency are introduced. On the basis of the fuzzy frequency, the fuzzy histogram over fuzzy colors is defined in section IV. An application to dominant color selection is presented in section $\mathrm{V}$ to illustrate the potential applications and performance of the new definition of fuzzy histogram. Some examples are showed in section VI and, finally, the main conclusions and future works are summarized in section VII.

\section{FUZZY MODELLING OF COLORS}

In this section, the notions of fuzzy color (section II-B) and fuzzy color space (section II-C) are introduced as an extension of the classical concepts of color and color space (section II-A). 


\section{A. Color and color spaces}

For representing colors, several color spaces can be used. In essence, a color space is a specification of a coordinate system and a subspace within that system where each color is represented by a single point. The most commonly used color space in practice is RGB because is the one employed in hardware devices (like monitors and digital cameras). It is based on a cartesian coordinate system, where each color consists of three components corresponding to the primary colors red, green, and blue.

Nevertheless, it is well known that RGB it is not always the most adequate space for color image analysis. Furthermore, the color components of this space do not have an intuitive interpretation according to the human perception of color. Instead, other color spaces based on human perception (HSI, HSV or HSL) seem to be a better choice [1]. In these perceptual spaces, the hue component $(\mathrm{H})$ represents the color tone (for example, red or blue), saturation (S) is the amount of color (for example, vivid red or pale red) and the third component (called intensity, value or lightness) is the amount of light (it allows to distinguish between a dark color and a light color). Therefore, if linguistic labels are needed for color description, this kind of color spaces are more suitable than RGB, linear combination of RGB (like CMY, $\mathrm{YCbCr}, \mathrm{YUV}$, etc.) or perceptually uniform color spaces (like CIELa* $b^{*}$, CIELuv, etc.).

\section{B. Fuzzy color}

In order to manage the imprecision in color description, we introduce the following definition of fuzzy color:

Definition 2.1: A fuzzy color $\widetilde{C}$ is a normalized fuzzy subset of colors.

As previously explained, colors can be represented as a triplet of real numbers corresponding to coordinates in a color space. Hence, a fuzzy color can be defined as a normalized fuzzy subset of points of a color space. From now on, we shall note XYZ a generic color space with components $\mathrm{X}, \mathrm{Y}$ and $\mathrm{Z}^{1}$, and we shall assume that a color space XYZ, with domains $D_{X}, D_{Y}$ and $D_{Z}$ of the corresponding color components is employed. This leads to the following more specific definition:

Definition 2.2: A fuzzy color $\widetilde{C}$ is a linguistic label whose semantics is represented in a color space XYZ by a normalized fuzzy subset of $D_{X} \times D_{Y} \times D_{Z}$.

Notice that it is possible to represent the same fuzzy color by means of different fuzzy subsets on different color spaces, provided they have the same expressive power. For example, a fuzzy color red could be represented as a fuzzy subset with semantics "approximately [255,0,0]" if we employ the RGB color space and also as a fuzzy subset with semantics "approximately $[0,1,128]$ " if we use the HSV space.

${ }^{1}$ Although we are assuming a three dimensional color space, the proposal can be easily extended to color spaces with more components.
As in the case of colors, that can be described by means of three precise component values, it is possible (though not obligatory) to define a fuzzy color by means of fuzzy subsets on the domain of each component. We introduce the following definition:

Definition 2.3: A fuzzy color component $\widetilde{C}_{X}$ (resp. $\widetilde{C}_{Y}$, $\widetilde{C}_{Z}$ ) is a linguistic label whose semantics is represented by a normalized fuzzy subset of $D_{X}$ (resp. $\left.D_{Y}, D_{Z}\right)$.

By using these concepts, a fuzzy color $\widetilde{C}$ can be defined and represented in practice by a triple $\left[\widetilde{C}_{X}, \widetilde{C}_{Y}, \widetilde{C}_{Z}\right]$, where $\widetilde{C}_{X}, \widetilde{C}_{Y}$, and $\widetilde{C}_{Z}$ are fuzzy color components of $\widetilde{C}$. This way, the fuzzy subset representing a fuzzy color can be obtained by combining the corresponding fuzzy color components in a suitable way. Thus, for every crisp color $[x, y, z]$, its membership degree to $\widetilde{C}$ is defined as

$$
\widetilde{C}(x, y, z)=\bigwedge\left\{\widetilde{C}_{X}(x), \widetilde{C}_{Y}(y), \widetilde{C}_{Z}(z)\right\}
$$

with $\bigwedge$ being a t-norm, usually the minimum.

\section{Fuzzy color space}

For extending the concept of color space to the case of fuzzy colors, and assuming a fixed color space XYZ, with $D_{X}, D_{Y}$ and $D_{Z}$ being the domains of the corresponding color components, the following definition is introduced:

Definition 2.4: A fuzzy color space $\widetilde{X Y Z}$ is a set of fuzzy colors that define a partition of $D_{X} \times D_{Y} \times D_{Z}$.

As in the case of single colors, one very convenient way of defining and representing a fuzzy color space is to employ a fuzzy space for each component. We introduce this idea in the following definition:

Definition 2.5: A fuzzy space over the component $X$ (resp. $Y, Z$ ) is a set of fuzzy color components that define a partition of $D_{X}\left(\right.$ resp. $\left.D_{Y}, D_{Z}\right)$.

This procedure has several advantages. First, less linguistic labels have to be defined. Second, we can represent and work with every component of the fuzzy color individually (this is specially interesting in perceptual color spaces like HSI where, for example, we could query for colors with red hue). Finally, the linguistic labels associated to fuzzy colors can be obtained by combining the corresponding linguistic labels of each component (for example, in the case of HSI color space, the color label "red-saturated-bright" is obtained by combining hue, saturation and intensity labels).

\section{FuZZy NATURALS AND FuZZY Rationals}

In order to obtain an histogram, which give us information about the frequency of each color in the image, we need to count the number of pixels having a particular color. In our case, we must count pixels having fuzzy colors. For this purpose we employ the concepts of fuzzy natural and fuzzy frequency. 
In this section we explain the definition of fuzzy naturals introduced in [5] and we use it as the basis to introduce a new definition of fuzzy frequency (section III-B). For defining fuzzy naturals the definition of cardinality of a fuzzy set will be used (section III-A)

\section{A. Fuzzy Cardinality}

The definition of fuzzy natural can be obtained directly from the definition of cardinality of a fuzzy set. In the crisp case, as it is well known, naturals are the possible cardinalities of crisp sets. The usual (in the sense of most employed) way to extend cardinality to the fuzzy case is the scalar sigma-count, that can defined for any fuzzy set $F$ with membership function $F: X \rightarrow[0,1]$ as

$$
s c(F)=\sum_{x \in X} F(x)
$$

However, the sigma-count is not a suitable measure of cardinality in many applications, as it has been recognized by several authors [13],[9]. In the particular problem of defining fuzzy naturals, sigma-count is counterintuitive, since $s c$ is not a natural number in general. Even if the result is a natural number, we can obtain the same result in very different situations, thus loosing information. This is the case if we consider two situations like having 100 pixels compatible with a color $\widetilde{C}$ to a degree 0.1 , and having 10 pixels compatible with $\widetilde{C}$ to a degree 1 ; in both cases, the result of the sigma count applied to the fuzzy set of pixels compatible with $\widetilde{C}$ is 10 .

On the basis of these problems, it is widely accepted that the best way to represent the cardinality of a fuzzy set is by means of a fuzzy subset of the naturals [8] [4]. In [8], Dubois and Prade stated the following:

"[...] it may sound paradoxical that ignoring the boundaries of a set, we may still be able to make a precise count of its elements, as scalar cardinality indices tend to suggest. Moreover, mathematicians may not feel very happy with a definition of cardinality yielding real numbers, while basically, the concepts of cardinality and integer are identical!"

Most of the approaches consider that such fuzzy subsets of the naturals must be convex. However, in [4] we showed that in some cases this is counterintuitive. Consider for example the fuzzy set given by $A=1 / x_{1}+0.5 / x_{2}+0.5 / x_{3}$. The cardinality of $A$ could be one (because $x_{1}$ belongs to $A$ for sure) or, if we relax our criterion to accept elements in $A$, the cardinality could be three (accepting $x_{2}$ and $x_{3}$ belong to $A$ as well). However, the cardinality cannot be two, since if $x_{2} \in A$ then $x_{3} \in A$ and vice versa. This way, the cardinality is not convex. In addition, this example illustrates that the sigma-count is not always a good measure since $\operatorname{sc}(A)=2$. In summary, $s c$ is not a good cardinality approach for the definition of fuzzy natural numbers since it is a real number and can give counterintuitive results.

Several authors [4], [15], [16] have pointed out that the possible cardinalities of a fuzzy set are the cardinalities of its $\alpha$-cuts, since these are the possible crisp representatives of the fuzzy set. In our previous example the possible cardinalities of $A$ are 1 or 3 since its possible $\alpha$-cuts are $\left\{x_{1}\right\}$ and $\left\{x_{1}, x_{2}, x_{3}\right\}$. In general, this is in accordance with the principle that there is a single membership scale for fuzzy sets, i.e., $F(x)=F\left(x^{\prime}\right)$ means that $x$ is $F$ just like $x^{\prime}$, so we cannot consider $x \in F$ and $x^{\prime} \notin F$ at the same time, and either both or none of them contribute to the cardinality of $F$.

As we have just seen, the sigma-count does not necessarily correspond to the cardinality of an $\alpha$-cut $(\operatorname{sc}(A)=2)$. On the contrary, there are two fuzzy cardinalities that comply with this idea, calculating the possible cardinalities of a fuzzy set as the cardinalities of its $\alpha$-cuts, and assigning to each cardinality a degree of representativity, as we detail in the following subsections:

1) Zadeh's first fuzzy cardinality: Zadeh introduced in 1979 a non-convex fuzzy measure for the cardinality of a fuzzy set $\mathrm{F}$ to be [23]

$$
\mu_{C A R D(F)}(k)=\sup \left\{\alpha|| F_{\alpha} \mid=k\right\}
$$

For example, for $A=1 / x_{1}+0.5 / x_{2}+0.5 / x_{3}$ we have $\mu_{C A R D(A)}=1 / 1+0.5 / 3$. In general, $\mu_{C A R D(\cdot)}$ is a fuzzy subset of $\mathbb{N}$ verifying it is normal, its core consists of one value only, and it is strictly decreasing in its support.

2) The Fuzzy Cardinality ED: In [4] the fuzzy cardinality of a fuzzy set $F$ is defined as

$$
E D(F)=\sum_{\alpha_{i} \in \Lambda(F)}\left(\alpha_{i}-\alpha_{i+1}\right) /\left|F_{\alpha_{i}}\right|
$$

where $\Lambda(F)=\left\{\alpha_{1}, \ldots, \alpha_{q}\right\}=\left\{F\left(x_{i}\right) \mid x_{i} \in \operatorname{supp}(F)\right\} \cup$ $\{1\}$ is the set of representative $\alpha$-cuts of $F$, with $1=\alpha_{1}>$ $\alpha_{2}>\cdots>\alpha_{q}>\alpha_{q+1}=0$. For example, $\Lambda(A)=\{1,0.5\}$ and $E D(A)=0.5 / 1+0.5 / 3$.

The measure ED is a probability distribution on $\mathbb{N}$. It is possible to show that the converse is also true, i.e., any probability distribution on $\mathbb{N}$ is the cardinality of some fuzzy set as given by ED. The proposition and the corresponding constructive proof, from [5], and an example from the same paper can be found in the appendix.

In [4] it is shown that $E D$ is the basic probability assignment of the possibility distribution $\mu_{C A R D(\cdot)}$, and hence both cardinalities are isomorphic. However, $E D$ has been shown to be more appropriate than $\mu_{C A R D(.)}$ when using the fuzzy cardinality in practice, for example in the evaluation of quantified sentences [6].

\section{B. Fuzzy naturals and fuzzy frequencies}

The idea that cardinality and natural numbers are basically identical was employed in [5] to define fuzzy natural numbers as follows:

Definition 3.1: A fuzzy natural $N$ is any probability dis- 
tribution on $\mathbb{N}$ of the form ${ }^{2}$

$$
N=\sum_{n_{i} \in \mathbb{N}} p_{i} / n_{i}
$$

with $\sum_{n_{i} \in N} p_{i}=1$.

As an example, let us consider the fuzzy natural

$$
N=0.2 / 0+0.6 / 2+0.2 / 4
$$

Having $N$ objects means that the number of objects is 0 with probability $0.2,2$ with probability 0.6 , and 4 with probability 0.2 .

In the context of histograms, this definition allows us to count the number of pixels that comply with a certain fuzzy color $\widetilde{C}$ as a fuzzy natural, corresponding to the cardinality of the fuzzy subset of pixels whose color is $\widetilde{C}$. However, we are interested in calculating the frequency of a fuzzy color $\widetilde{C}$ in the set of pixels as an estimation of the probability of that color in the image. This is usually obtained as the quotient between the number of pixels with color $\widetilde{C}$ and the number of pixels in the image. For that purpose, we can easily extend the notion of fuzzy natural to that of fuzzy rational number as follows:

Definition 3.2: Let $N$ be a fuzzy natural number and let $p \in \mathbb{N}$ with $p>0$. Then $N / p$ is the fuzzy rational number

$$
N / p=\sum_{q_{i}=\left(n_{i} / p\right) \mid n_{i} \in \mathbb{N}} p_{i} / q_{i}
$$

with $p_{i}=N\left(n_{i}\right)$.

As a consequence of the definition, $N / p$ is a probability distribution on the set of rationals $\{n / p \mid n \in \mathbb{N}\} \subseteq \mathbb{Q}$. Notice that this definition is not intended to characterize every possible fuzzy rational number, but just the subset of them obtained by dividing a fuzzy natural by a natural number not equal to 0 .

In this paper we are interested in fuzzy rationals $N / p$ such that $p=\max \{n \in \operatorname{support}(N)\}$, i.e., fuzzy rationals such that $\operatorname{support}(N / p) \subseteq[0,1]$. This kind of fuzzy rationals can be employed in order to obtain the frequency of a fuzzy color $\widetilde{C}$ as follows: let $N_{\widetilde{C}}$ be the fuzzy natural corresponding to the cardinality of the fuzzy subset of pixels that verify $\widetilde{C}$, and let $n$ be the number of pixels in the image. Then, the frequency of $\widetilde{C}$ in the image is the fuzzy rational $N_{\widetilde{C}} / n$.

This fuzzy frequency can be understood as a (fuzzy) estimation of the probability of $\widetilde{C}$ in the image, i.e., a fuzzy probability understood as a probability distribution on the rational interval $[0,1][24]$. Notice that we are dealing with two kind of probabilities here:

- First, the (precise, crisp) probabilities that form the probability distribution $N_{\widetilde{C}} / p$.

- Second, the (imprecise, fuzzy) probability of $\widetilde{C}$ in the image, i.e, the probability that a pixel taken at random

${ }^{2}$ Notice that we are using in the definition of $N$ the usual notation $\sum$ for fuzzy sets on a discrete referential, while in $\sum_{n_{i} \in N} p_{i}=1, \sum$ means addition. from the image is painted with the color $\widetilde{C}$. This is estimated by the fuzzy rational $N_{\widetilde{C}} / p$.

\section{FuZZY Histograms}

We define a fuzzy histogram as follows:

Definition 4.1: Let $\widetilde{X Y Z}$ be a fuzzy color space. A fuzzy histogram is a function $h$ that assigns a fuzzy rational to every fuzzy color in $\widehat{X Y Z}$, i.e.,

$$
h(\widetilde{C})=N_{\widetilde{C}} / n
$$

with $N_{\widetilde{C}}$ a fuzzy natural and $\operatorname{support}\left(N_{\widetilde{C}} / n\right) \subseteq[0,1]$.

In practice, the histogram for a given image with $n$ pixels is obtained by calculating $N_{\widetilde{C}}$ as the fuzzy natural representing the cardinality of the fuzzy subset of pixels with color $\widetilde{C}$, noted $I_{\widetilde{C}}$, from which the fuzzy rational $N_{\widetilde{C}} / n$ is obtained as in equation 7 .

Notice that this definition extends to the fuzzy case the idea of histogram as a probability distribution on the set of colors (or some crisp quantization). Histograms can be also defined as functions that assign a count, given by a natural number, to every color. This alternative can be extended to the fuzzy case by replacing the fuzzy rational $N_{\widetilde{C}} / n$ by the fuzzy natural $N_{\widetilde{C}}$ in definition 4.1.

Fuzzy histograms are an accurate description of the fuzzy probability that a pixel has a color $\widetilde{C}$, for every color in a fuzzy color space. However, if this is the final information to be provided to a user, it is convenient to summarize it so that it is easier to understand. Of course, any possible summary causes a loss of information. We can consider several options as a summary of the fuzzy frequency of a fuzzy color:

- Linguistic summary of the fuzzy rationals in $[0,1]$. In this option, a triangular fuzzy number (in the classical sense of "possibility distribution on the set or real numbers") $\mathcal{R}_{\widetilde{C}} / n$ is calculated as an approximation of the fuzzy rational $N_{\widetilde{C}} / n$. The number is obtained by approximation techniques as the number that yields a maximum accomplishment degree with $N_{\widetilde{C}} / n$ with minimum entropy. This technique is described in [2].

- The interval of rational numbers $[a, b]$ with

$$
\begin{aligned}
a & =\min \left\{i \in \operatorname{support}\left(N_{\widetilde{C}} / n\right)\right\} \\
b & =\max \left\{i \in \operatorname{support}\left(N_{\widetilde{C}} / n\right)\right\}
\end{aligned}
$$

- The rational number with higher probability in the probability distribution $N_{\widetilde{C}} / n$. Notice that this number, being an scalar, is obtained from the cardinality of an $\alpha$-cut of $I_{\widetilde{C}}$, and in this sense is coherent with the ideas explained at the beginning of section III.

- The rational number $l / n$ with $l$ being the cardinality of the 0.5 -cut of the fuzzy subset of pixels $I_{\widetilde{C}}$. This scalar value is the definition of approximation of the cardinality of a fuzzy set proposed by Ralescu [15]. In addition, if the fuzzy color space is a partition in the sense of Ruspini, the 0.5 -cut corresponds to a crisp partition of the color space, and the values obtained 


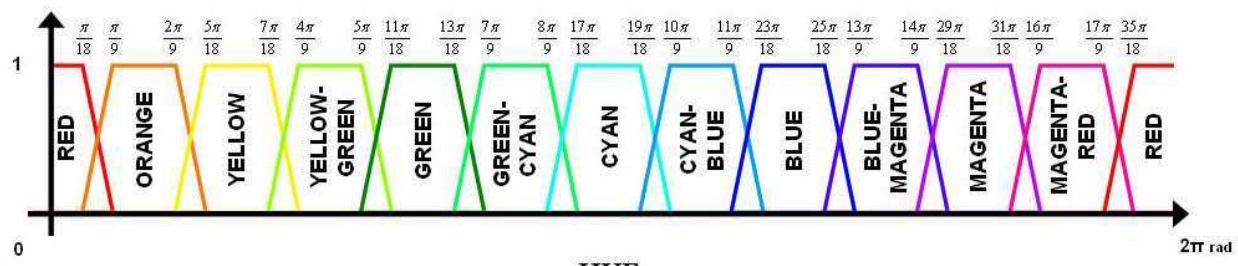

HUE
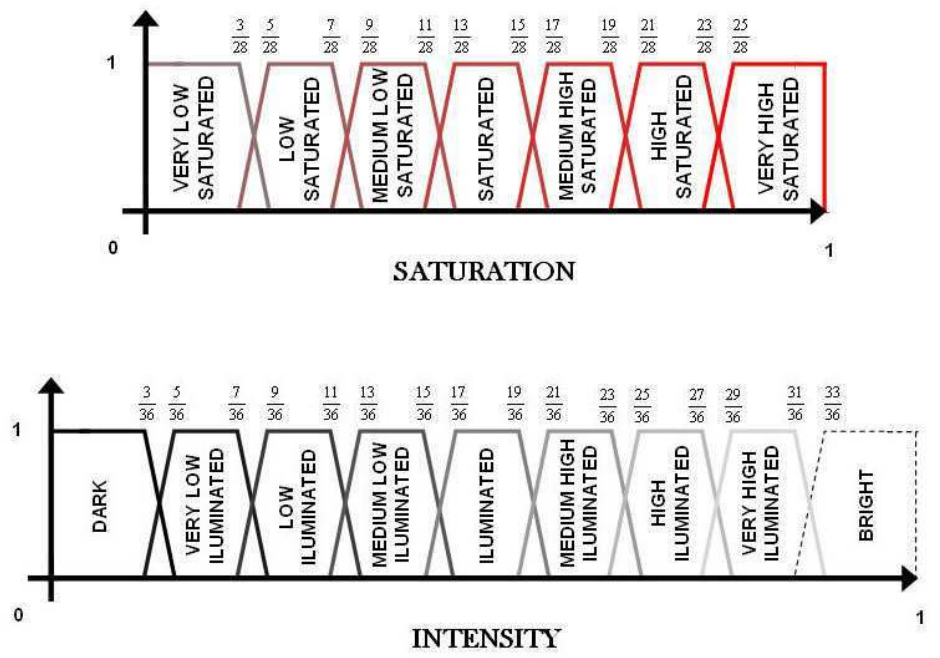

Fig. 1. The fuzzy HSI color space used in the experiments.

for all the fuzzy colors correspond to a crisp histogram based on this crisp partition.

Let us remark once more that the latter are different ways to obtain a summary that can be useful for a better understanding of the fuzzy histogram by an user, at the cost of loosing information. However, if the histogram is just an intermediate step in a calculation, we can extend the operations to the fuzzy case by operating on $\alpha$-cuts and then summarizing the final result (see appendix). This option preserves all the information until the final step. The option of extending the operations to the fuzzy case by operating on one of the previously suggested summaries discards many information, and it is reasonable in our opinion only when time restrictions force us to obtain a less accurate, but faster, calculation.

Notice that we are not suggesting that the calculations performed by $\alpha$-cuts are necessarily slow; the idea is that performing a certain operation in every $\alpha$-cut takes more time than performing the same operation on a single scalar approximation. Depending on the time expended by each operation and the number of $\alpha$-cuts employed (the latter can be fixed in order to obtain an approximate, but more accurate, result; a typical value is 20 ), we may choose one or another alternative.

\section{Application to Dominant Color SElection}

In this section we illustrate the potential applications and performance of the new definition of fuzzy histogram by applying it to select a set of dominant fuzzy colors. Dominant colors arise as a powerful tool for describing the representative colors in an image. In fact, they are a very efficient tool for retrieving images in large databases.

Intuitively, a color is dominant to the extent it appears frequently in a given image. Therefore, the use of fuzzy histograms comes up as a suitable tool for finding the dominant fuzzy colors.

Let $h$ be a fuzzy histogram for a color image on a fuzzy color space $\widehat{X Y Z}$. Given a color $\widetilde{C}$, its fuzzy frequency is given by $h(\tilde{C})=N_{\widetilde{C}} / n$ with $N_{\widetilde{C}} / n=$ $\sum_{q_{i}=\left(n_{i} / n\right), n_{i} \in \mathbb{N}} p_{i} / q_{i}$ being a fuzzy rational number in the sense of definition 3.2. We define the degree of dominance of the fuzzy color $\widetilde{C}$ as

$$
\operatorname{Dom}(\widetilde{C})=\sum_{q_{i}>T} p_{i}
$$

with $T \in(0,1]$ being a threshold for selecting significant frequencies.

As set of dominant fuzzy colors we will select those ones with higher dominant degree (we can fix a number of 
dominant colors or select those ones which dominant degrees are greater than a threshold)

\section{RESUlts}

In this section we show the results obtained by applying the proposed technique to several examples. Firstly, the fuzzy histogram will be calculated over several images. Secondly, these histograms will be used to select dominant fuzzy colors.

In our experiments, the HSI color space have been used. To define the fuzzy color space $\widetilde{H S I}$ we have employed the fuzzy spaces for hue, saturation, and intensity proposed in [3] that are shown in figure 1. This proposal used as reference the Munsell color space [20] which divided in 12, 7 and 9 intervals the hue, saturation, and intensity respectively. Each one of these intervals are fuzzified using a trapezoidal function to define the memberships functions. As result we obtain a fuzzy partition in the sense of Ruspini. ${ }^{3}$

Figure 2 shows two images, the first one containing eight colors, each one compatible with degree 1 with a fuzzy color obtained by combining the fuzzy saturation label "High saturated" and the fuzzy intensity label "Illuminated", with eight different fuzzy hue labels, specifically all the hue labels except "Yellow-Green", "Cyan", "Blue-Magenta", and "Red" in figure 1. In the second one we have four colors compatible with the same saturation and intensity labels to degree 1 . Each one of these four colors is compatible to a degree 0.5 with two of the eight fuzzy hue labels mentioned before. In both images, pixels are equidistributed among colors.

In both images, the set of fuzzy colors with probability greater than 0 is the set of eight fuzzy colors mentioned above. It is easy to see that the sigma-count histogram is the same for both images, specifically the probability is $1 / 8$ for each fuzzy color. However, the perception of the colors that appear in the image and the corresponding frequencies is very different in both images.

On the contrary, the histogram based on our definition of fuzzy frequencies yield a different result in each case. In the first one, since we are in a crisp case, the result is the same provided by the sigma-count histogram, i.e., probability $1 / 8$ (given as a probability distribution $1 /(1 / 8)$ ) for each fuzzy color. On the contrary, our approach yields for the second image a fuzzy histogram where, for each one of the eight color, the fuzzy probability is represented by the probability distribution $0.5 / 0+0.5 /(1 / 4)$. This is in accordance with our intuition since each fuzzy color is compatible to a degree 0.5 with one of the colors that appear in the image and, as a consequence, we have a probability 0.5 that the fuzzy label appears in the image, and the same probability that it does not appear. Hence, the same probabilities are assigned to the value $1 / 4$ in the first case, and 0 in the second case.

Figures 3(B) to 3(I) show the fuzzy histogram for image 3(A). For plotting the histogram, a graph has been drawn for each intensity label. The $\mathrm{X}$ axis of these graphs shows

${ }^{3}$ The problem of non-representability in semichromatic (undefined hue) and achromatic (undefined hue and saturation) is taken into account in the sense proposed in [3]

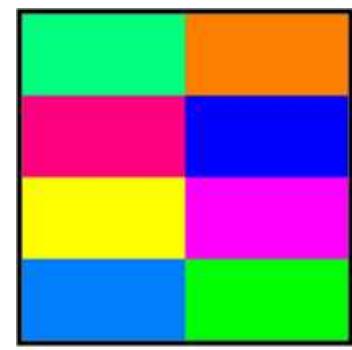

A

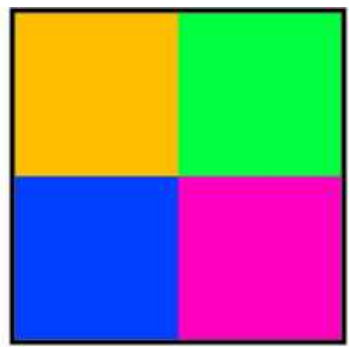

$\mathbf{B}$
Fig. 2. Multicolored images. (A) Image containing eight colors, each one compatible with degree 1 with a fuzzy color (B) Image containing four colors, each one compatible with degree 0.5 with a fuzzy color

the color labels ordered by hue and, within a given hue, by saturation. The $\mathrm{Y}$ axis shows the support of $h(\widetilde{C})$. More specifically, the graph plots pairs $\left(\widetilde{C}, q_{i}\right)$ where $q_{i}$ are the crisp frequencies that appear in the probability distribution $N_{\widetilde{C}}$. In addition, a dotted line is drawn between the maximum and minimum values $\mathrm{a}$ and $\mathrm{b}$ of the support, $a=\min \{i \in$ $\left.\operatorname{support}\left(N_{\widetilde{C}} / p\right)\right\}$ and $b=\max \left\{i \in \operatorname{support}\left(N_{\widetilde{C}} / p\right)\right\}$. This dotted line shows the interval $[a, b]$ that corresponds to one of the ways of summarizing $h(\widetilde{C})$, as explained in section IV. The values $N_{\widetilde{C}}\left(q_{i}\right)$ are not displayed.

We have compared the results obtained in this and other images with the crisp histogram for a crisp partition obtained as the set of 0.5-cuts of the fuzzy colors. Though the latter is a summary and many information is lost, we have found that higher (resp. lower) crisp frequencies in the crisp histogram correspond to higher (resp. lower) values in the support of fuzzy frequencies and, in this sense, the results are coherent with what is expected. However, the fuzzy histogram contains much more information since, contrary to the crisp one, it reflects the imprecision associated to the frequencies.

The fuzzy histogram shows greater frequency values in the support of the most frequent fuzzy colors in the image. For example, the highest values correspond to the fuzzy colors Black (Dark intensity) and the brown-like colors [Orange,Medium-Low-Saturated,Illuminated] and [Orange,Saturated,Illuminated], these three fuzzy colors corresponding to the background. The next colors in terms of high frequency are those of the teapot.

Figure 4 shows the degree of dominance of those colors with degree of dominance greater than 0 for the image in Figure 3(A). The dominance has been calculated by using a threshold $T=0.015$. The results are coherent with the information in the fuzzy histogram. With respect to our perception of dominance in the image, we must take into account that the fuzzy color space employed in the chromatic area of the HSI color space is a equidistributed fuzzy partition. However, as pointed out by several authors, we tend to employ a non-equidistributed partition in which a single fuzzy color covers approximately the support of the fuzzy colors with hue "Cyan", "Cyan-Blue" and "Blue". 

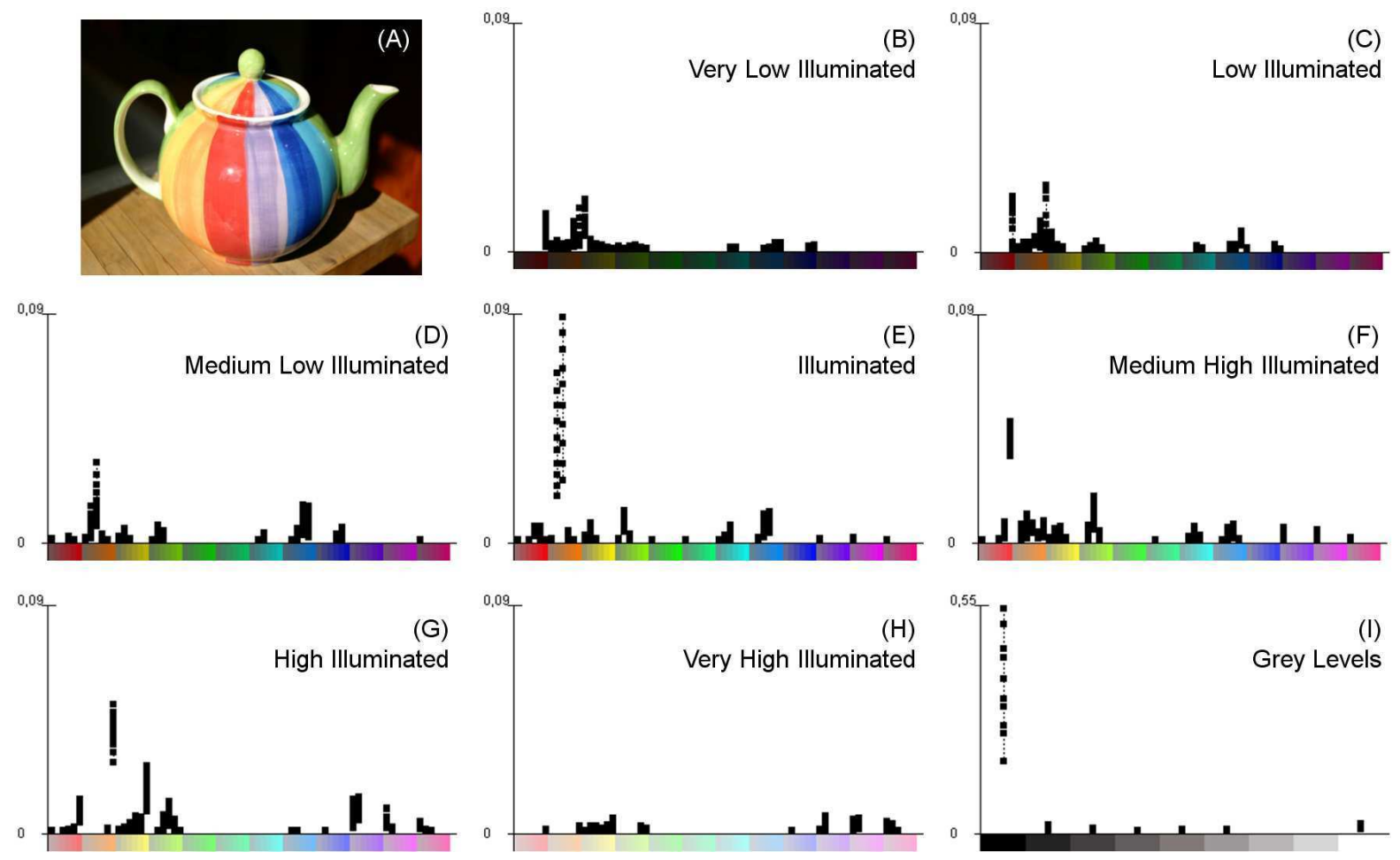

Fig. 3. (A): color image. (B)-(H): fuzzy histograms for colors with different intensity labels except "Dark" and "Bright". In each histogram, the 12x6 colors ("Very low saturated" colors, i.e., fuzzy grey levels, are not considered) are ordered first by hue and then by saturation. (I): Fuzzy colors corresponding to fuzzy grey levels.

Something similar happens with the fuzzy colors with hue labels around "Green". The consequence in this example is that the colors we see as blue are distributed between the fuzzy colors with hue "Cyan", "Cyan-Blue" and "Blue", so the degree of dominance of what we see as blue is maybe less than expected. On the contrary, if we consider a fuzzy color space with a label "Blue" covering the support of the three above mentioned blue-based colors, the dominance of the new label would be similar to other fuzzy colors with "Red", "Orange" or "Yellow" fuzzy hue.

\section{CONCLUSIONS AND FUTURE WORK}

In this paper a novel definition of fuzzy histogram has been proposed. Firstly, a formalization of the concept of fuzzy color and fuzzy color space has been presented. Secondly, the occurrences of a given fuzzy color have been counted by means of a new definition of fuzzy natural number. On the basis of these fuzzy naturals, a new definition of fuzzy frequency has been proposed, where a rational number has been represented by means of a probability distribution on $\mathbb{R}$.

The proposal improves the classical sigma-count based histogram. In fact, our fuzzy histogram represents the imprecision of the count itself, contrary to sigma-count that summarizes the counting in a single number.
The performance of the proposal has been shown with several images, introducing some examples where histograms based on sigma-count are not a suitable representation of the amount of pixels painted with each color. In addition, we have illustrated the potential applications of the fuzzy histogram by applying it to the problem of dominant fuzzy colors selection.

As future work, classical operations on histograms will be extended to the fuzzy case, applying the techniques and operations to problems like information retrieval, among others.

\section{APPENDIX}

The representation of a fuzzy rational (resp. a fuzzy natural) is a summary of the information given by the $\alpha$ cut representation by using the associated basic probability assignment, assuming that from the point of view of the cardinality the objects that are assigned the appropriate degrees are unimportant. Both representations are equivalent, and we can easily obtain one of them from the other:

Proposition A.1[5]: Let $R$ be a probability distribution on $\mathbb{N}$, then there exists at least one fuzzy set $F_{R}$ over a large enough universe $X$ such that $R=E D\left(F_{R}\right)$.

Proof: Let $\operatorname{supp}(R)=\left\{i_{1}, \ldots, i_{m}\right\}$ with $i_{1}<i_{2}<$ $\cdots<i_{m}$ and let $i_{0}=0 . F_{R}$ can be obtained by the following algorithm: 


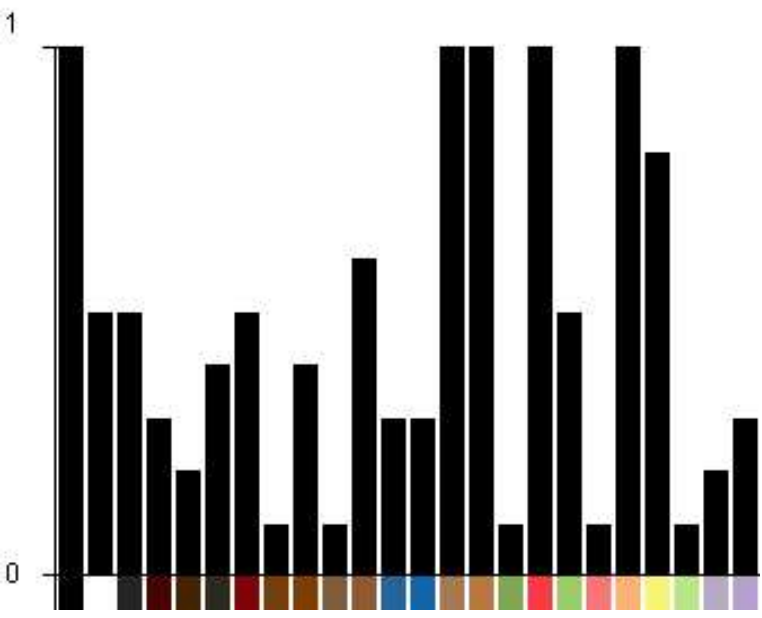

Fig. 4. Degree of dominance of fuzzy colors in the image of figure 3(A). Only fuzzy colors with non-zero dominance are shown.

1) $F_{R} \leftarrow \emptyset ; j \leftarrow 1 ; \mu=1$

2) If $i_{1}=0$ then $j \leftarrow 2 ; \mu \leftarrow 1-R\left(i_{1}\right)$

3) While $j \leq m$

a) Add $i_{j}-i_{j-1}$ new elements with degree $\mu$ to $F_{R}$

b) $\mu \leftarrow \mu-R\left(i_{j+1}\right)$

c) $j \leftarrow j+1$

It is easy to check that $\Lambda\left(F_{R}\right)=\{1\} \cup\left\{1-r_{p} \mid 1 \leq p<m\right\}$ with $r_{p}=\sum_{1 \leq k \leq p} R\left(i_{k}\right)$. Also, let $\Lambda\left(F_{R}\right)=\left\{\alpha_{1}, \ldots, \alpha_{m}\right\}$ with $\alpha_{1}>\alpha_{2}>\cdots>\alpha_{m}$. Then $\left|\left(F_{R}\right)_{\alpha_{j}}\right|=i_{j}$ and $\alpha_{j}-\alpha_{j+1}=R\left(i_{j}\right)$. Hence

$$
E D\left(F_{R}\right)=\sum_{1 \leq j \leq m} R\left(i_{j}\right) / i_{j}=R .
$$

Example: $\quad$ Let $\quad R=0.12 / 1+0.6 / 2+0.28 / 4$, then we have $\operatorname{supp}(R)=\left\{i_{1}=1, i_{2}=2, i_{3}=4\right\}$, $\Lambda\left(F_{R}\right)=\{1,0.88,0.28\}$, and the algorithm will add $i_{1}-i_{0}=1$ new element to $F_{R}$ with degree $\mu=1$, $i_{2}-i_{1}=1$ new element with degree 0.88 , and $i_{3}-i_{2}=2$ new elements with degree 0.28 . Hence, $F_{R}$ will be of the form

$$
F_{R}=1 / x_{1}+0.88 / x_{2}+0.28 / x_{3}+0.28 / x_{4}
$$

Operations on fuzzy naturals as introduced in [5] follow the idea of performing the corresponding crisp operations on the values associated to $\alpha$-cuts and finally obtaining degrees associated to each possible result as the addition of the probabilities associated to the $\alpha$-cuts where the result appears. This is in accordance with the interpretation of the corresponding operations in terms of sets. For example, addition of fuzzy naturals $N_{1}$ and $N_{2}$ gives as result the cardinality of the union of the sets $F_{N_{1}}$ and $F_{N_{2}}$ by assuming that the support of them is disjoint [5]. In [5] it is shown that the resulting arithmetic operations and ranking of fuzzy naturals have very good and intuitive properties.

\section{REFERENCES}

[1] A. Del Bimbo. Visual Information Retrieval. Morgan Kaufmann Publishers, 2001

[2] I. Blanco, D. Sánchez, J.M. Serrano, and M.A. Vila. A new proposal of aggregation functions: The linguistic summary. In T. Bilgiç, B. De Baets, and O. Kaynak, editors, Fuzzy Sets and Systems - Proceedings IFSA'03, LNAI 2715, pages 127-134. Springer, 2003.

[3] J. Chamorro-Martínez, J.M. Medina, C. Barranco, E. Galán-Perales, and J.M. Soto-Hidalgo. Retrieving images in fuzzy object-relational databases using dominant color descriptors. Fuzzy Sets and Systems, 158(3):312-324, February 2007.

[4] M. Delgado, M.J. Martín-Bautista, D. Sánchez, and M.A. Vila. A probabilistic definition of a nonconvex fuzzy cardinality. Fuzzy Sets and Systems, 126(2):41-54, 2002.

[5] M. Delgado, M.J. Martín-Bautista, D. Sánchez, and M.A. Vila. Fuzzy integers: Representation and arithmetic. In Proceedings of IFSA'05, 2005.

[6] M. Delgado, D. Sánchez, and M.A. Vila. Fuzzy cardinality based evaluation of quantified sentences. International Journal of Approximate Reasoning, 23:23-66, 2000.

[7] A. Doulamis and N. Doulamis. Fuzzy histograms for efficient visual content representation: application to content-based image retrieval. In IEEE International Conference on Multimedia and Expo, pages 893896, Aug 2001.

[8] D. Dubois and H. Prade. Fuzzy cardinality and the modeling of imprecise quantification. Fuzzy Sets and Systems, 16:199-230, 1985.

[9] D. Dubois, H. Prade, and T. Sudkamp. A discussion of indices for the evaluation of fuzzy associations in relational databases. In T. Bilgic, B. De Baets, and O. Kaynak, editors, Fuzzy Sets and Systems - Proceedings IFSA'03, LNAI 2715, pages 111-118. Springer, 2003.

[10] J. Han and Kai-Kuang. Fuzzy color histogram and its use in color image retrieval. IEEE Transactions on Image Processing, 11(8):944952, 2002.

[11] L. Hildebrand and M. Fathi. Knowledge-based fuzzy color processing. IEEE. Tran. on Systems, Man and Cybernetics. Part C, 34(4):499-505, November 2004

[12] G. Louverdis, I. Andreadis, and Ph. Tsalides. New fuzzy model for morphological colour image processing. In IEEE Proc. Vis. Image Signal Processing, volume 149, June 2002.

[13] M.J. Martín-Bautista, D. Sánchez, M.A. Vila, and H.L. Larsen. Measuring effectiveness in fuzzy information retrieval. In H.L. Larsen, J. Kacprzyk, S. Zadrozny, T. Andreasen, and H. Christiansen, editors, Proceedings FQAS 2000, Advances in Soft Computing Series, pages 396-402. Springer-Verlag, 2000.

[14] T. Mitsuishi, N. Kayaki, and K. Saigusa. Color construction using dual fuzzy system. In IEEE Int. Sym. Computational Intelligence for Measurement Systems and Applications, pages 136-139, 2003.

[15] D. Ralescu. Cardinality, quantifiers and the aggregation of fuzzy criteria. Fuzzy Sets and Systems, 69:355-365, 1995.

[16] D. Rocacher and P. Bosc. The set of fuzzy rational numbers and flexible querying. Fuzzy Sets and Systems, 155(3):317-339, 2005.

[17] S. Romani, P. Sobrebilla, and E. Montseny. Obtaining the relevant colors of an image through stability-based fuzzy color histograms. In IEEE International Conference on Fuzzy Systems, volume 2, pages 914-919. St. Louis, Missouri (USA), May 2003.

[18] T.A. Runkler. Fuzzy histograms and fuzzy chi-squared tests for independence. In IEEE International Conference on Fuzzy Systems, volume 3, pages 1361-1366, July 2004.

[19] J.C. Russ. The Image Processing Handbook. CRC Press and IEEE Press, third edition, 1999.

[20] S.J. Sangwine and R.E.N. Horne. The colour image processing handbook. Chapman and Hall, 1998.

[21] M. Seaborn, L. Hepplewhite, and J. Stonham. Fuzzy colour category map for the measurement of colour similarity and dissimilarity. Pattern Recognition, 38(4):165-177, 2005.

[22] N. Sugano. Color-naming system using fuzzy set theorical approach. In IEEE Int. Conference on Fuzzy Systems, pages 81-84, 2001.

[23] L. A. Zadeh. A theory of approximate reasoning. Machine Intelligence, 9:149-194, 1979.

[24] L.A. Zadeh. Fuzzy probabilities. Information Processing and Management, 20(3):363-372, 1984.

[25] H. Zhu, H. Zhang, and Y. Yu. Deep into color names: Matching color descriptions by their fuzzy semantics. LNAI 4183, pages 138-149, 2006 